\title{
The diameter and Laplacian eigenvalues of directed graphs
}

\author{
Fan Chung \\ University of California, San Diego \\ La Jolla, CA 92093-0112
}

Submitted: Feb 13, 2006; Accepted: Feb 14, 2006; Published: Feb 22, 2006

Mathematics Subject Classification: 05C20

\begin{abstract}
For undirected graphs it has been known for some time that one can bound the diameter using the eigenvalues. In this note we give a similar result for the diameter of strongly connected directed graphs $G$, namely

$$
D(G) \leq\left\lfloor\frac{2 \min _{x} \log (1 / \phi(x))}{\log \frac{2}{2-\lambda}}\right\rfloor+1
$$

where $\lambda$ is the first non-trivial eigenvalue of the Laplacian and $\phi$ is the Perron vector of the transition probability matrix of a random walk on $G$.
\end{abstract}

\section{Introduction}

For an undirected graph $G$ on $n$ vertices, we can upper bound the diameter $D(G)$ by using eigenvalues of the Laplacian $[1,2]$ as follows.

$$
D(G) \leq\left\lfloor\frac{\log (n-1)}{\log \frac{\lambda_{n-1}+\lambda_{1}}{\lambda_{n-1}-\lambda_{1}}}\right\rfloor+1
$$

where $0=\lambda_{0} \leq \lambda_{1} \leq \cdots \leq \lambda_{n-1}$ denote the eigenvalues of the Laplacian of $G$.

A natural question is to see if it is feasible to extend such relations to directed graphs. In a directed graph, the distance and diameter can be naturally defined: The distance from a vertex $u$ to a vertex $v$ is the length of a shortest directed path from $u$ to $v$. A directed graph is strongly connected if for any two vertices $u$ and $v$, there is a directed path from $u$ to $v$. The diameter of a strongly connected directed graph is defined to be the maximum distance among pairs of vertices. If a directed graph is not strongly connected, its diameter is taken to be infinity. 
In a recent paper [4], the Laplacian of a directed graph was defined. The eigenvalues of this Laplacian turn out to be quite useful for capturing various isoperimetric properties of directed graphs. For example, the spectral gap of the Laplacian can be used to bound the mixing rate of random walks in directed graphs. The eigenvalues of the Laplacian were also used to establish a general Cheeger inequality. In this note, we will establish the following diameter bound by using the spectral gap of the Laplacian for a directed graph.

Theorem 1. For a strongly connected graph $G$, the diameter $D(G)$ of $G$ satisfies

$$
D(G) \leq\left\lfloor\frac{2 \min _{x} \log (1 / \phi(x))}{\log \frac{2}{2-\lambda}}\right\rfloor+1
$$

where $\lambda$ is the first non-trivial eigenvalue of the Laplacian and $\phi$ is the Perron vector of the random walk on $G$.

We will define our terminology in Section 2 and give a proof for Theorem 1 in Section 3. In the last section we consider diameter bounds for Cayley graphs and give some other remarks.

\section{Preliminaries}

In this section we define our terminology. Suppose $G$ is a directed graph with vertex set $V(G)$ and edge set $E(G)$. For a vertex $u$, the out-degree of $u$, denoted by $d_{u}$, is the number of edges leaving $u$.

A random walk on $G$ is defined by its transition probability matrix $P$, where for vertices $u$ and $v$, the probability of moving from $u$ to $v$ is given by

$$
P(u, v)= \begin{cases}\frac{1}{d_{u}} & \text { if }(u, v) \text { is an edge } \\ 0 & \text { otherwise. }\end{cases}
$$

Thus, $P(u, v)>0$ if and only if $(u, v)$ is an edge, and $\sum_{v} P(u, v)=1$.

In the remainder of this paper, a random walk on $G$ means the typical random walk as defined above unless specified otherwise. Since

$$
P 1=1
$$

where 1 denotes the all 1 s vector (as a column vector), the spectral radius is 1 . The Perron-Frobenius Theorem implies that the transition probability matrix $P$ of a strongly connected directed graph has a unique (up to scaling) left eigenvector $\phi$ with $\phi(v)>0$ for all $v$ achieving the spectral radius, i.e.,

$$
\phi P=\phi .
$$

By scaling, we choose $\phi$ satisfying

$$
\sum \phi(v)=1
$$

THE ELECTRONIC JOURNAL OF COMBINATORICS 13 (2006), \#N4 
(Here we treat $\phi$ as a row vector.) We call $\phi$ the Perron vector of $P$, which is its stationary distribution when the aperiodic condition is satisfied (i.e., the GCD of all cycle lengths is $1[4])$. Unlike undirected graphs, the Perron vector can have entries that are exponentially small.

The Laplacian of a directed graph $G$ is defined by

$$
\mathcal{L}=I-\frac{\Phi^{1 / 2} P \Phi^{-1 / 2}+\Phi^{-1 / 2} P^{*} \Phi^{1 / 2}}{2}
$$

where $\Phi$ is a diagonal matrix with entries $\Phi(v, v)=\phi(v)$ and $P^{*}$ denotes the conjugated transpose of $P$. The Laplacian $\mathcal{L}$ has eigenvalues $0=\lambda_{0} \leq \lambda_{1} \leq \ldots \leq \lambda_{n-1}$, where the first non-trivial eigenvalue $\lambda=\lambda_{1}$ satisfies the following [4]:

$$
\lambda=\inf _{\substack{f \\ f \Phi \mathbf{1}=0}} \frac{\sum_{u \rightarrow v}|f(u)-f(v)|^{2} \phi(u) P(u, v)}{2 \sum_{v}|f(v)|^{2} \phi(v)} .
$$

\section{Proof of the main theorem}

To show that a directed graph $G$ has diameter $D$, it is enough to show that for some matrix $M$ and for all vertices $x$ and $y$, we have

$$
M^{D}(x, y)>0
$$

provided that for $x \neq y$, we have $M(x, y)=0$ if $(x, y) \notin E(G)$.

We will use the following fact from [4]. For the sake of completeness we include a proof which is short and different from the one in [4].

Lemma 1. Suppose that a strongly connected directed graph $G$ has transition probability matrix $P$ and a lazy random walk $\mathcal{P}=(I+P) / 2$. Then the matrix $M=\Phi^{1 / 2} \mathcal{P} \Phi^{-1 / 2}$ satisfies

$$
\frac{\|f M\|^{2}}{\|f\|^{2}} \leq 1-\frac{\lambda}{2}
$$

for all vectors $f$ satisfying $f \Phi^{1 / 2} \mathbf{1}=0$. 
Proof. We consider $g$ with $g \Phi^{1 / 2}=f$ and we have

$$
\begin{aligned}
\frac{\|f M\|^{2}}{\|f\|^{2}} & =\frac{\sum_{y}\left|\sum_{\substack{x \rightarrow y \\
x \rightarrow y}}(g(x)+g(y)) \phi(x) P(x, y)\right|^{2} \phi(y)^{-1}}{4 \sum_{y}|g(y)|^{2} \phi(y)} \\
& \leq \frac{\sum_{y} \sum_{\substack{x \rightarrow y \\
x \rightarrow y}}|g(x)+g(y)|^{2} \phi(x) P(x, y)}{4 \sum_{y}|g(y)|^{2} \phi(y)} \\
& =1-\frac{\sum_{y} \sum_{x \rightarrow y}|g(x)-g(y)|^{2} \phi(x) P(x, y)}{4 \sum_{y}|g(y)|^{2} \phi(y)} \\
& \leq 1-\frac{\lambda}{2} .
\end{aligned}
$$

The first inequality follows by the Cauchy Schwarz inequality $\left(\left(\sum_{x} a_{x} b_{x}\right)^{2} \leq \sum_{x} a_{x}^{2} \sum_{x} b_{x}^{2}\right.$ with $\left.a_{x}=|g(x)+g(y)| \sqrt{\phi(x) P(x, y)}, b_{x}=\sqrt{\phi(x) P(x, y)}\right)$ and (2) (i.e., $\sum_{x} \phi(x) P(x, y)=$ $\phi(y))$. The next equality is easily verified noting that $|a+b|^{2}+|a-b|^{2}=|a|^{2}+|b|^{2}$ and then using $(2)$ and $(1)$ (i.e., $\sum_{y} P(x, y)=1$ ). The final inequality follows from $(3)$.

As an immediate consequence, we have for $F$ satisfying $f \Phi^{1 / 2} \mathbf{1}=0$

$$
\frac{\left\|f M^{k}\right\|^{2}}{\|f\|^{2}} \leq\left(1-\frac{\lambda}{2}\right)^{k}
$$

Proof of Theorem 1. For a fixed vertex $x$, we define $f_{x}$ by

$$
f_{x}(y)= \begin{cases}1-\phi(x) & \text { if } y=x \\ -\sqrt{\phi(x) \phi(y)} & \text { otherwise. }\end{cases}
$$

Clearly, $f_{x}$ satisfies $f_{x} \Phi^{1 / 2} \mathbf{1}=0$. Note that $1 \Phi^{1 / 2}$ is a left eigenvector of $M$ associated with the eigenvalue 1 . Now, for any positive integer $k$ and any two vertices $x$ and $y$ of $G$, we consider

$$
\begin{aligned}
\left|M^{k}(x, y)-\sqrt{\phi(x) \phi(y)}\right| & =\left|f_{x} M^{k}(y)\right| \\
& \leq\left\|f_{x} M^{k}\right\| \\
& <\left(1-\frac{\lambda}{2}\right)^{k / 2}
\end{aligned}
$$

since $\left\|f_{x}\right\|=\sqrt{1-\phi(x)}<1$. 
Suppose that we choose $k$ satisfying

$$
k=\left\lfloor\frac{2 \min _{x} \log (1 / \phi(x))}{\log \left(\frac{2}{2-\lambda}\right)}\right\rfloor+1 .
$$

Then we have

$$
\begin{aligned}
M^{k}(x, y) & \geq \sqrt{\phi(x) \phi(y)}-\left(1-\frac{\lambda}{2}\right)^{k / 2} \\
& \geq \min _{z} \phi(z)-\left(1-\frac{\lambda}{2}\right)^{k / 2} \\
& >0 .
\end{aligned}
$$

This shows that the diameter $D(G)$ satisfies $D(G) \leq k$ completing the proof.

\section{Concluding remarks}

Of special interest is the case for directed Cayley graphs whose vertex set is a group $\Gamma$ and the directed edges are generated by a subset $B$ of elements of $\Gamma$ as $\{(x, b x): x \in \Gamma, b \in B\}$. For a directed Cayley graph, the condition of strong connectivity is equivalent to the assumption that $B$ is a generating set. Furthermore, the stationary distribution of a directed Cayley graph is the uniform distribution. Therefore, we have the following:

Corollary 1. Suppose that a directed Cayley graph $G$ has vertex set $\Gamma$ with $|\Gamma|=n$ and edges of $G$ are generated by a generating set $B$. Then the diameter $D(G)$ of $G$ satisfies

$$
D(G) \leq\left\lfloor\frac{2 \log n}{\log \frac{2}{2-\lambda}}\right\rfloor+1
$$

where $\lambda$ is the first non-trivial eigenvalue of the Laplacian.

To compare the result for directed graphs with the known diameter bound for undirected graphs, we consider known explicit constructions of Ramanujan graphs. By replacing each undirected edge by two directed edges of opposite directions, the associated directed Ramanujan graph has the same eigenvalues. Corollary 1 yields an upper bound within a factor of 4 of the bound for the undirected case.

We have now seen that the eigenvalues of the Laplacian can be used to control the diameter of the graph. It is also known that the eigenvalues of the Laplacian are closely related to the convergence of random walks in a directed graph. In [4] it is shown that in a strongly connected directed graph, the first non-trivial Laplacian eigenvalue $\lambda$ provides an upper bound for the rate of convergence for the lazy random walk as follows:

$$
2 \Delta_{T V}(t) \leq(1-\lambda / 2)^{t / 2} \max _{y} \phi(y)^{-1 / 2}
$$


Where $\Delta_{T V}(t)$ is the total variation distance after $t$ steps, i.e.,

$$
\begin{aligned}
\Delta_{T V}(t) & =\max _{A \subset V(G)} \max _{y \in V(G)}\left|\sum_{x \in A}\left(P^{t}(y, x)-\phi(x)\right)\right| \\
& =\frac{1}{2} \max _{y \in V(G)} \sum_{x \in V(G)}\left|P^{t}(y, x)-\phi(x)\right| .
\end{aligned}
$$

Is it possible to find a similar lower bound for the rate of convergence in terms of $\lambda$ ?

Another important consequence of the Laplacian eigenvalues is its relationship with the Cheeger constant of a directed graph [4]. It is of interest to see what further properties

of directed graphs can be controlled by the Laplacian eigenvalues. Can we bound the average distance in terms of the Laplacian eigenvalues, for example?

\section{Acknowledgement}

The author wishes to thank Steve Butler for useful comments. This research is supported in part by NSF Grants DMS 0100472 and ITR 0205061.

\section{References}

[1] F. Chung, Diameters and eigenvalues, J. of Amer. Math. Soc. 2 (1989), 187-196.

[2] F. Chung, V. Faber and T. A. Manteuffel, An upper bound on the diameter of a graph from eigenvalues associated with its Laplacian, SIAM J. Discrete Math. 7 (1994), 443-457.

[3] F. Chung, Spectral Graph Theory, AMS Publications, 1997.

[4] F. Chung, Laplacians and the Cheeger inequality for directed graphs, Annals of Combinatorics 9 (2005), 1-19. 